\title{
Research on Innovative Design Method of In-wheel Jet Flying Car-Dunhuang Feitian
}

\author{
Yunbo Ji \\ Academy of Fine Arts and Art Design, Luoyang Normal College, Luoyang City, Henan Province ,471934, China
}

\begin{abstract}
The car has been invented for more than a century. In the 21 st century, new cars will realize the function of land and sky bidirectional driving. The author designed a flying concept car, powered by a wheel jet engine, named Dunhuang Feitian. He won the second prize in the Second China University Art Competition. The car is equipped with a "turbine ram engine" inside the four hubs. The aerodynamic layout is designed in a cross shape. The wheel engine jets downwards, which can flexibly adjust the flight attitude. The author did not sacrifice industrial design aesthetics in order to highlight flight performance. He borrowed the beautiful curve contours of Dunhuang Feitian in the body design, designed the car to be streamlined, and equipped with a four-axis stabilization system in safety configuration. performance. In addition, this article also predicts the future development of automobiles, and provides comfortable, environmentally friendly, green solutions to alleviate the increasingly serious traffic congestion problems, and has a broad application prospect.
\end{abstract}

\section{$1 \quad$ History of flying cars}

\subsection{The Origin of Flying Cars}

In 1907, the French Breguet brothers invented the four-rotor helicopter, and backward manipulation led to poor flight stability.

\subsection{Bionic concept flight vehicle}

In 1990, Aeromobil broke the traditional thinking, sought inspiration from birds in nature, and invented a flying car that mimics the principle of biological flight[1].

\subsection{AirQuad One Flying Motorcycle}

Neva Aerospace exhibited an "AirQuadOne vertical take-off and landing motorcycle" at the Paris Aviation Exhibition, which is the world's first solo flying motorcycle.

\subsection{Pop.up flight car}

Audi plans to build a "Flying City of the Future" and exhibits a "pop.up flying car" at the Geneva Motor Show, with a 5-level gear and four electric wing engines.

\subsection{China TF-X Flying Car}

"Corresponding author's e-mail :493102441@qq.com
China's Geely Automobile Co., Ltd. uses "Volvo" safety technology to develop two types of flying cars, Terrafugia and TF-X. The former requires a short runway for take-off, and the latter uses folded wings to take off and land vertically to a high altitude without a runway.

\section{Mechanical design flaws make flying cars look ugly}

\subsection{Today 's flying cars, sacrificing beautiful design in order to improve flight performance}

At present, flying cars can run, take off, and cruise in short distances. In appearance, it is similar to a traditional aircraft (glider). In order to meet the aerodynamics, the beautiful design is sacrificed. The shape is weird and has no aesthetics.

\subsection{Flying cars put their engines on the outside of the body and couldn't get rid of the plane}

The "AirCar" flying car has four seats and four doors, and all flying mechanical parts are exposed to the outside [2]. In land mode, a car can fold its huge wings in seconds. In air mode, it can cruise at a speed of $250 \mathrm{~km} /$ $\mathrm{h}$ at 7620 meters.

\subsection{Flying cars are bulky, inflexible and take up a lot of space}

\section{TEL:13838838132}


The "Müller Aerocar" flying car is equipped with four propellers that can change direction. The volume is huge, the long wings are hidden under the body, and the area is very large and bulky.

\section{Wheel jet engine design, not only maintain beautiful, but also have good flight performance}

\subsection{Hiding the jet engine in the wheels keeps the flying car in good shape (figure 1)}

The Dunhuang Feitian flying car cleverly conceals the jet engine in the wheels, refuses to reduce the design aesthetics for flight performance, and maintains a streamlined body. With a size of 5919/1810 / 1519mm and a wheelbase of $2919 \mathrm{~m}$, it reaches the same level of luxury car "Bentley Continental GT".

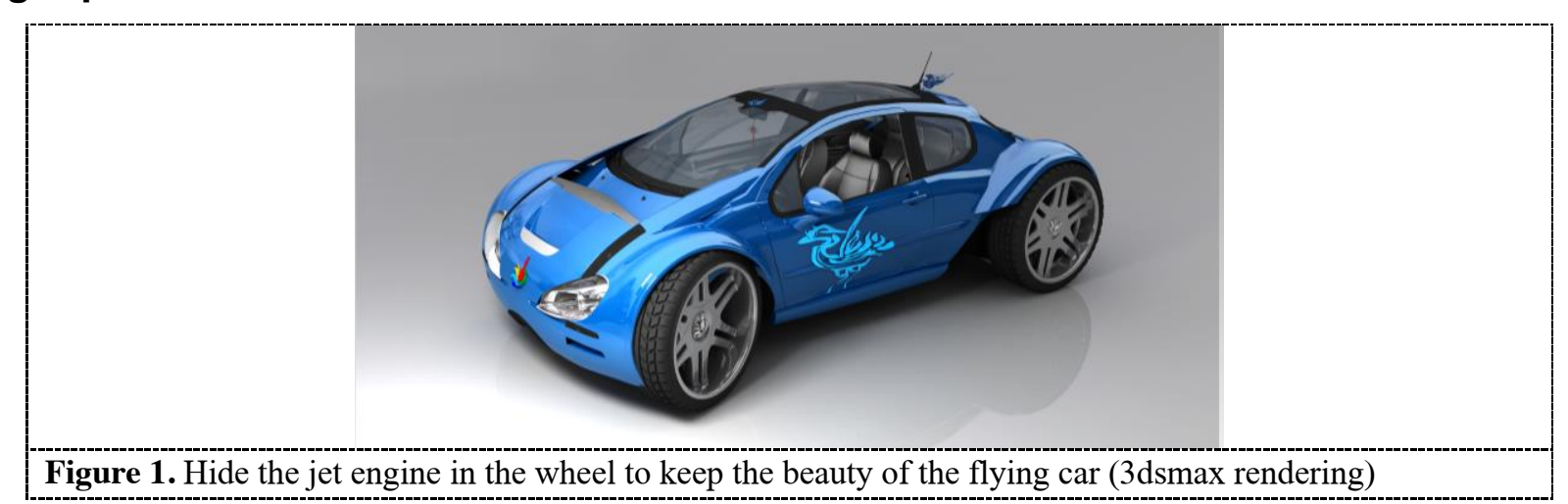

\subsection{Four-axis mechanical power design solves the contradiction between "beautiful appearance" and "flying performance"}

When the car is docked, it is an elegant and handsome sports car. When the car is driving, the jet engine hidden in the wheels does not need to be extended. When the car is flying, the wheels are extended outward and the jet is jetted downward. The vehicle has no extra bracket parts. No wings are needed, the interior space is spacious, and the storage is abundant, which does not take up space.

\subsection{Drivers use $\mathrm{Al}$ cloud intelligence, big data internet, and 5G communication to control wheel four-axle engines}

The driver monitors the vehicle speed and wind speed through the DSP body nerve chain sensor, uses AI cloud intelligence to automatically identify coordinates, and uses 5G communication for unmanned driving. The on-board computer CPU can perform massive data calculations. In car monitoring, a 720-degree panoramic camera is used to remove obstacles. The navigation is "China Beidou, GPS, Galileo".

\section{$4 \quad$ Telescopic robotic arm, U-shaped vent, retro-style center console}

\subsection{Sky blue car paint symbolizes passion and vitality}

The car is covered with sky blue paint, symbolizing passion, vitality, and technology. On the front side of the car, an "inner recess" air duct is designed, which is matched with the symmetrical spoiler and echoes with the dual exhaust ports of the rear lights to make the whole vehicle seamless. The four wheels of the car are equipped with thick metal alloy wheels, which are tightly supported by a telescopic robotic arm, which is aerodynamic and shows mechanical beauty.

\subsection{The design of the front face of the car is a combination of "U-shaped hidden grille" and LED}

The air intake grille of the air vent adopts a "U-shaped" design. The LED headlights are composed of two circular car lights of different sizes gradually moving closer to the center. They look like smiling faces of people, are very dynamic, and have unique machinery. Beauty feeling.

\subsection{Retro design of center console}

The central control platform is not decorated with technological materials, but is wrapped in dark leather, expressing elegant retro beauty. In the middle of the central control platform is a "penetrating instrument display bar", inlaid with walnut wood, high-grade solid wood texture and advanced mechanical structure, forming a strong sense of impact. At the bottom right of the central control platform is an electronic gearshift, with manual and automatic operation. The driver will step on the accelerator and experience the "push back feeling" of flying off the ground and hear the "jet sound and engine noise".

\section{4. "Telescopic Wheel Mechanical Structure" supports large-sized "copper-aluminum composite wheels", highlighting the beauty of technology}

All four wheels are equipped with 8.0T "turbo ramjet engines", which can pulse air at high altitude to generate 818 horsepower thrust. The wheels are equipped with thick copper-aluminum composite metal wheels with a radius of $680 \mathrm{~mm}$, more than half the height of the vehicle. 
The engine tightly supports the wheel hub with a telescopic "robotic arm". The gearbox controls "takeoff, lift, cruise, and back", and solves the three problems of "vertical lifting, folding fixed wings, and exposed spiral wings", showing the beauty of science and technology.

\section{The application of "flying apsaras art" in wheel design of dunhuang frescoes in China}

\subsection{Modern Industrial Design with "Hetu Luoshu" and "Dunhuang Feitian"}

The author is the inheritor of the intangible cultural heritage of "World Cultural Heritage-Longmen Grottoes". $\mathrm{He}$ is good at painting oriental art and has created many award-winning works. The work "Longmen Grottoes Landscape Art" hand-carved "Ancient Dunhuang Fresco Flying" (Figure 2), in 2019, won the five-yearly "Thirteenth Chinese Art Exhibition", in 2018, the painting "He "Tuluoshu. Aerospace. Flying" won the "Sixth China Youth Art Exhibition". When designing the wheels and body of a flying car, he borrowed the "flying sky" from the ancient Dunhuang murals in China, and painted "Hetu Luoshu, Dragon Horse, Phoenix" on the door. The blue car looks like an ancient " Taishan Moyu "perfectly combines Dunhuang art and modern design on the Silk Road (Figure 3).
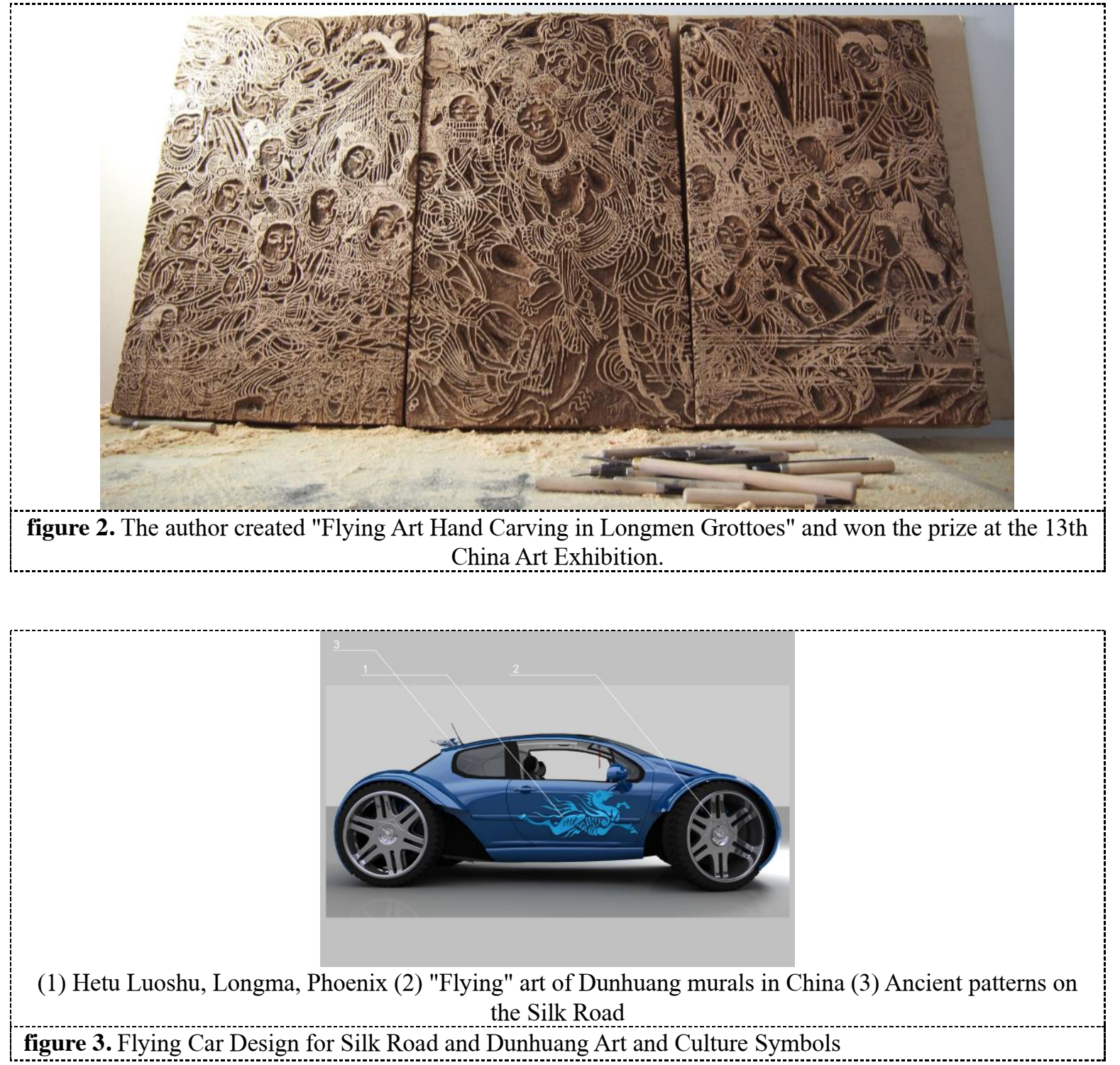

Auxiliary power is "ultra-high-speed electric-driven fan

\subsection{From take-off to cruise, the two steps of mechanical operation demonstrate aerodynamic aesthetics}

\subsubsection{Step 1: Take off by jet .}

The auxiliary power is used to accelerate before the working speed can be reached to ignite the ram engine. blade engine", which is connected to 24 sets of gears through ring bearings. It rotates at high speed to generate powerful airflow, push up the car, and the mechanical support arm controls the direction of the wheel. Figure 4).

\subsubsection{The second step: high-altitude stamping.}

Use the oncoming airflow to decelerate after entering the 
engine, increase the static pressure, enter the diffuser, ignite in the combustion chamber, and generate super

high-speed hot air.

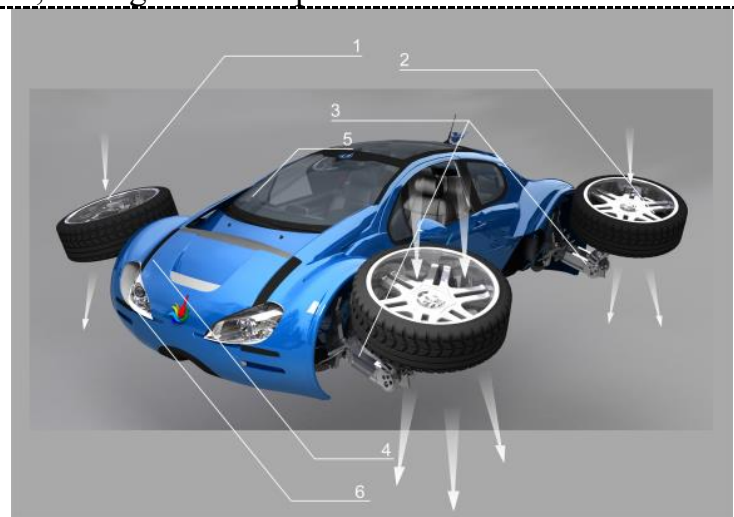

(1) Turbo ramjet engine (2) Four-axis balance (3) Hub support arm (4) U-shaped intake grill (5) Windshield (6) LED

figure 4. The mechanical structure of the automobile's flight engine, the operation process of the turbojet (3dsmax modeling effect diagram).

In the future, in order to solve the problem of urban traffic congestion, there will be public flying cars that can

6 Inspiration of the innovative design concept of "wheel jet flying car" to future car design

\subsection{The mainstream trend of flying cars in the future will be "smarter, more beautiful and smaller"} take hundreds of people. However, when dissatisfied with people, many empty seats will be wasted and resources will be occupied, so designers will avoid designing oversized The car is designed as a smart, beautiful and convenient new vehicle for "shared independent cabin flying vehicle, single-person flying scooter" (Figure 5).

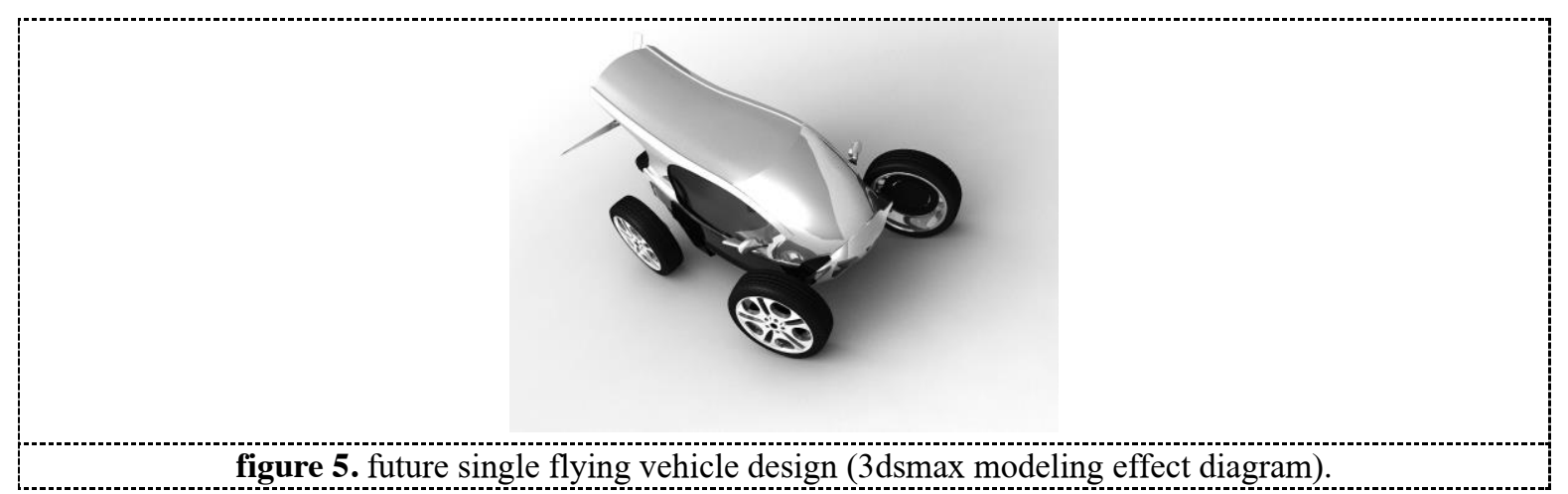

figure 5. future single flying vehicle design (3dsmax modeling effect diagram).

\subsection{In the future, new flying cars inventing "maglev and antigravity engines"}

In the future, with the breakthrough of theoretical physics, complex gears will disappear in mechanical manufacturing, the sound of rumbling gasoline engines will be replaced by quiet graphene batteries, metals with memory functions will be popularized, cars will be printed and manufactured by $3 \mathrm{D}$ printers, and glass The car window is a holographic transparent screen, which is transparent when viewed from the inside to the outside of the car, as if floating in the air. Anti-gravity engines will be invented, cars will get rid of the constraints of gravity, can distort space and time, use "zero-point energy" to provide unlimited power, shuttle in "planetary magnetic lines-wormholes", fly near the speed of light without inertia, high dimensions In the space jump, people eliminated the steering wheel and used "quantum communication-telepathy" to consciously control the car to achieve long-distance material transmission and reach interstellar travel (Figure 6). 


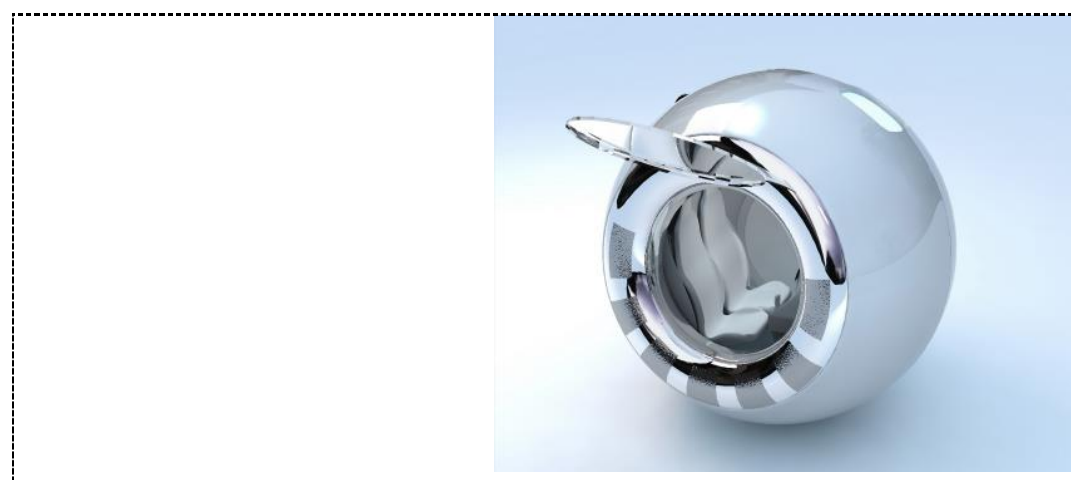

figure 6. Future anti-gravity vehicle design to implement interstellar travel (3dsmax modeling effect diagram).

\section{References}

1. Tao Han. (2014) Research on the development direction of mechanical design, manufacture and automation. Henan Science and Technology, 181:108-109

2. Jichao Li. (2008) Art of industrial design creation and manufacture. Digital and Design, 281:81-85

3. Li Zhang, Wenxia Zhang. (2001) Application of three-dimensional printing materials for the manufacture of automotive parts. Technology outlook, 197:96-99 